\title{
Amorphous vs crystalline exciton blocking layers at the anode interface in planar and planar-mixed heterojunction organic solar cells
}

Cite as: Appl. Phys. Lett. 104, 213304 (2014); https://doi.org/10.1063/1.4879839

Submitted: 22 April 2014 . Accepted: 14 May 2014 . Published Online: 28 May 2014

Stefan Grob, Mark Gruber, Andrew N. Bartynski, Ulrich Hörmann, Theresa Linderl, Mark E. Thompson, and Wolfgang Brütting
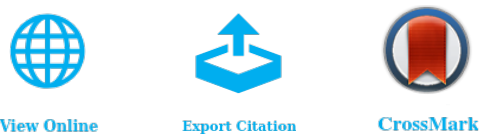

\section{ARTICLES YOU MAY BE INTERESTED IN}

Small molecular organic photovoltaic cells with exciton blocking layer at anode interface for improved device performance

Applied Physics Letters 99, 153302 (2011); https://doi.org/10.1063/1.3650472

Two-layer organic photovoltaic cell

Applied Physics Letters 48, 183 (1986); https://doi.org/10.1063/1.96937

A hybrid planar-mixed tetraphenyldibenzoperiflanthene $/ \mathrm{C}_{70}$ photovoltaic cell

Applied Physics Letters 102, 073302 (2013); https://doi.org/10.1063/1.4793195

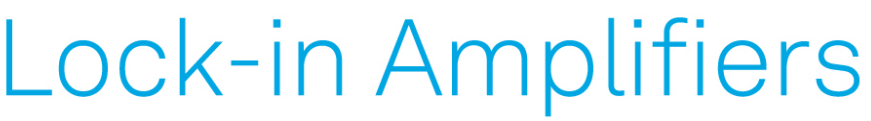

... and more, from DC to $600 \mathrm{MHz}$

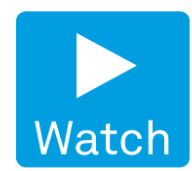




\title{
Amorphous vs crystalline exciton blocking layers at the anode interface in planar and planar-mixed heterojunction organic solar cells
}

\author{
Stefan Grob, ${ }^{1, a)}$ Mark Gruber, ${ }^{1}$ Andrew N. Bartynski, ${ }^{2}$ Ulrich Hörmann, ${ }^{1}$ Theresa Linderl, ${ }^{1}$ \\ Mark E. Thompson, ${ }^{2}$ and Wolfgang Brütting ${ }^{1, b)}$ \\ ${ }^{1}$ Institute of Physics, University of Augsburg, 86135 Augsburg, Germany \\ ${ }^{2}$ Departement of Chemical Engineering and Department of Chemistry, University of Southern California, \\ Los Angeles, California 90089, USA
}

(Received 22 April 2014; accepted 14 May 2014; published online 28 May 2014)

\begin{abstract}
We compare the gain in power conversion efficiency (PCE) achieved by inserting either amorphous or crystalline exciton blocking layers at the anode interface for planar (PHJ) and planar-mixed heterojunction (PM-HJ) organic solar cells based on Tetraphenyldibenzoperiflanthene and fullerenes. For PHJ devices, there is a gain of more than $37 \%$ for both types of blocking layers, mainly due to an increase in photocurrent, indicating that this gain can be solely ascribed to the exciton blocking effect. A templating effect as proposed in literature for crystalline blocking layers cannot be affirmed. On the contrary, it is shown that there is a connection between the choice of acceptor $\left(\mathrm{C}_{60} / \mathrm{C}_{70}\right)$ and the blocking effect on the anode side. Moreover, we can show that also for PM-HJ devices a remarkable efficiency enhancement is possible. The insertion of suitable blocking layers at the anode interface can alter the effective work function and thus the open-circuit voltage, leading to a maximum PCE of 5.8\% in single junction cells. C 2014 AIP Publishing LLC. [http://dx.doi.org/10.1063/1.4879839]
\end{abstract}

The use of exciton blocking layers in organic donoracceptor solar cells is well established, however, so far the focus has mainly been on the cathode. There, such blocking layers are mandatory as they prevent the penetration of subsequently evaporated cathode material (e.g., Al, Ag) into the active layer. In addition, the application of materials like bathocuproine $\left(\mathrm{BCP}^{1,2}\right)$ or bathophenanthroline $\left(\mathrm{BPhen}^{3,4}\right)$ also enhances the efficiency of organic solar cells by suppressing exciton-quenching at the metal-organic interface. At the opposite contact, it is also common to insert a buffer layer consisting of, e.g., $\mathrm{MoO}_{x}$ or poly(ethylenedioxythiophene):poly(styrenesulfonate) (PEDOT:PSS) between anode which is usually built up on indium tin oxide (ITO) - and donor material. This is done because ITO alone, due to its insufficient work function, cannot act as the desired hole-selective contact leading to high leakage currents. But it is usually neglected that, like ITO itself, these buffer layers are also exciton quenchers due to their quasi-metallic nature.

For planar heterojunction (PHJ) organic solar cells, it has already been shown that this recombination channel can be suppressed by inserting either crystalline ${ }^{5}$ or amorphous blocking layers, ${ }^{6}$ resulting in higher power conversion efficiencies (PCE) by notably elevating the short-circuit current density $\left(J_{\mathrm{SC}}\right)$, while leaving the values of open-circuit voltage $\left(V_{\mathrm{OC}}\right)$ and fill factor $(\mathrm{FF})$ almost unchanged. It was also suggested that the use of crystalline blockers as a nanostructured template could increase the area of the donor/acceptor interface, which would further enhance $J_{\mathrm{SC}}$, indicating that crystalline blockers are more favorable. ${ }^{5}$

In this work, we seek to clarify the influence of morphology by comparing blocking layers consisting of either crystalline diindenoperylene (DIP) or amorphous $\mathrm{N}, \mathrm{N}^{\prime}$-bis (naphthalen-1-yl)-N, $\mathrm{N}^{\prime}$-bis(phenyl)-2,2'-dimethylbenzidine

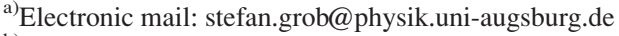

${ }^{b)}$ Electronic mail: wolfgang.bruetting@physik.uni-augsburg.de
}

$(\alpha-\mathrm{NPD})$ in planar as well as in planar-mixed heterojunction (PM-HJ) devices. The blocking materials are selected based on the alignment of their energy levels related to highest occupied molecular orbital (HOMO) and lowest unoccupied molecular orbital (LUMO) of the donor material. To achieve hole transport and efficient exciton blocking simultaneously, the ionization potential must be smaller (or at least similar), while its energy gap has to be wider compared to the donor. To emphasize the blocking effect, a highly absorbing donor material is chosen. Tetraphenyldibenzoperiflanthene $\left(\mathrm{DBP}^{7}\right)$ has already been proven to form efficient solar cells in combination with fullerenes providing high PCEs up to $8.1 \%{ }^{8}$ Its ability to absorb efficiently is due to the horizontal orientation of the molecules, which enables a strong coupling between the incident light and the transition dipole moment, which is aligned along the long axis of the molecule. ${ }^{9}$ As electron acceptor material, we mainly used the fullerene $\mathrm{C}_{60}$. However, some selected cells were fabricated using the stronger absorbing fullerene $\mathrm{C}_{70}$ to achieve a maximum PCE of $5.8 \% \pm 0.2 \%$ in single junction cells.

Fig. 1 shows the used organic materials with their energy levels and absorption spectra. The PEDOT:PSS derivative HIL1.3 was obtained from Clevios (Germany), $\alpha$-NPD and DBP from Lumtec (Taiwan), DIP from S. Hirschmann (Univ. Stuttgart, Germany), and BCP from Sigma-Aldrich. HIL1.3 was spin-coated from aqueous dispersion and subsequently dried at $125^{\circ} \mathrm{C}$ for $30 \mathrm{~min}$. All other materials were evaporated in UHV $\left(<5 \times 10^{-7}\right.$ millibars $)$ at $0.5 \AA / s$. Current-voltage $(\mathrm{J}-\mathrm{V})$ characteristics were recorded using a source measure unit (Keithley $236 \mathrm{SMU}$ ) under illumination with a solar simulator (Oriel $300 \mathrm{~W}$ with AM 1.5 G filters) in a glovebox system with nitrogen atmosphere. The illumination intensity was approved by a calibrated silicon reference cell (RERA systems, PV Measurement Facility, Radboud University Nijmegen, area $1 \times 1 \mathrm{~cm}^{2}$ ). Incident photon-to-current efficiency (IPCE) 


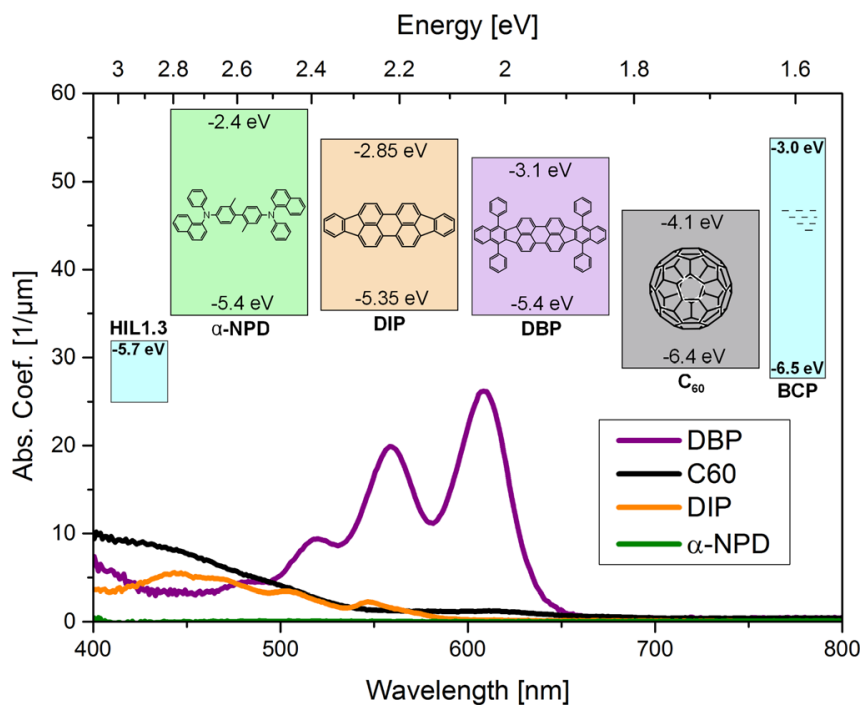

FIG. 1. Schematic drawing of molecular formulae and energy levels of $\alpha$-NPD, DIP, DBP, and $\mathrm{C}_{60}$. Energy values of organic materials are taken from literature. ${ }^{10,11}$ Moreover the absorption characteristics of donor, acceptor, and blocking materials are shown, with DBP featuring the by far most dominant absorption, while DIP and $\alpha$-NPD absorb only weakly.

measurements were carried out using a monochromatized Xe arc lamp as light source and lock-in detection.

Generally, the architecture of the PHJ solar cells is ITO $(140 \mathrm{~nm}) / \mathrm{HIL} 1.3(45 \mathrm{~nm}) /$ blocking layer $(\mathrm{y} \mathrm{nm}) / \mathrm{DBP}$ $(15 \mathrm{~nm}) /$ fullerene $(45 \mathrm{~nm}) / \mathrm{BCP}(5 \mathrm{~nm}) / \mathrm{Al}(100 \mathrm{~nm})$, i.e., the only variables are the blocking layer material and the thickness of that layer. DIP is chosen to form the crystalline blocking layer, exhibiting exceptionally high structural order in evaporated thin films. ${ }^{12}$ The DIP molecule, consisting of seven benzene and two cyclopentadiene rings, also forms the backbone of the DBP molecule, resulting in similar optical spectra. Nevertheless, there are significant differences. The DBP molecule has one further benzene ring on each side, increasing the $\pi$-electron system and thus leading to a redshift of absorption and therefore a smaller energy gap. Moreover, it features four additional, rotatable phenyl groups, changing the molecular orientation within the layer and by that the absorption coefficient (Fig. 1). Nevertheless, both requirements for effective exciton blocking are met: The energy gap for DIP is wider, as absorption measurements reveal, while UPS measurements show identical HOMO-offsets for $\mathrm{DIP} / \mathrm{C}_{60}$ and $\mathrm{DBP} / \mathrm{C}_{60}$ as required for hole transport to the anode. Due to its high order in evaporated thin films with large exciton diffusion lengths of up to $100 \mathrm{~nm},{ }^{13}$ DIP is also used as donor material in organic solar cells, yielding exceptionally high fill factors of nearly $75 \% .{ }^{14}$ However, the almost upright standing alignment of the DIP molecules leads to weak absorption and therefore a much smaller $J_{\mathrm{SC}}$ compared to DBP. This weak absorption is also advantageous in blocking layers, otherwise parasitic absorption can occur and the gain in current could not solely be ascribed to decreasing quenching effects. We excluded the impact of DIP absorption by varying the thickness of the blocking layer from 3 to $21 \mathrm{~nm}$ in $3 \mathrm{~nm}$ steps receiving almost identical values for $J_{\mathrm{SC}}$ (Fig. 2(a)). This result leads to the assumption that $3 \mathrm{~nm}$ of DIP already forms a (nearly) closed layer, which is in accordance with investigations,


FIG. 2. (a) $J$-V-characteristics, (b) IPCE curves and (c) solar cell parameters vs thickness of the DIP blocking layer. The architecture of the PHJ solar cells is $\operatorname{ITO}(140 \mathrm{~nm}) / \mathrm{HIL} 1.3(45 \mathrm{~nm}) / \mathrm{DIP}(\mathrm{x} \mathrm{nm}) / \mathrm{DBP}(15 \mathrm{~nm}) / \mathrm{C}_{60}(45 \mathrm{~nm}) /$ $\mathrm{BCP}(5 \mathrm{~nm}) / \mathrm{Al}(100 \mathrm{~nm})$. For reasons of clarity, some curves were omitted in (a) and (b).

revealing the tendency of DIP to grow in Stranski-Krastanov mode on various substrates. ${ }^{15,16}$ Compared to the reference without blocking layer, the gain in $J_{\mathrm{SC}}$ is between a minimum of $24 \%$ (3 nm DIP) and a maximum of $30 \%(6 \mathrm{~nm}$ DIP), staying nearly constant for higher thicknesses of the DIP layer. Moreover, also the values for $V_{\mathrm{OC}}$ (continuously) and FF (at first) show a small increase (Fig. 2(c)). This slight but continuous gain in $V_{\mathrm{OC}}$ for thicker blocking layers is an additional effect of the reduced recombination, ${ }^{17}$ whereas the fill factor increases from $69 \%(0 \mathrm{~nm})$ to a maximum of $72 \%(6 \mathrm{~nm})$ but then decreases again down to its initial value $(21 \mathrm{~nm})$ due to transport issues.

The PCE increases from $2.8 \%$ for the reference up to $3.8 \%$ for the best cell in this series containing a $6 \mathrm{~nm}$ DIP blocking layer, an improvement of more than $37 \%$. Furthermore, the similarity of the $J$ - $V$-characteristics of the 
compared solar cells leads to the conclusion that a possible template effect is not relevant. Due to pronounced island growth for higher DIP thicknesses, the root mean square roughness for the DIP/DBP interface increases, however, this effect does not propagate to the DBP/fullerene interface, where it could lead to enhanced exciton dissociation and thus a higher $J_{\mathrm{SC}}$. As well, AFM images and XRD measurements (not shown) do not reveal any signs of changed morphology and structure of the DBP layer. Even for DIP grown at elevated temperatures $\left(\mathrm{T}_{\text {Substrate }}=100{ }^{\circ} \mathrm{C}\right)$, which leads to an enhanced lateral crystallinity of the DIP layer, ${ }^{18}$ no changes in $J_{\mathrm{SC}}$ can be observed (open symbols in Fig. 2(a)). Therefore, the by far most dominant effect for the gain in $J_{\mathrm{SC}}$ is reduced exciton quenching at the HIL1.3/organic interface, which is also supported by IPCE measurements (Fig. 2(b)), revealing that the increment is mainly at wavelengths $(\lambda)$ between $500 \mathrm{~nm}$ and $650 \mathrm{~nm}$, where the maximum absorption of DBP occurs (Fig. 1). In the main absorption region of $\mathrm{C}_{60}(400 \mathrm{~nm}<\lambda<500 \mathrm{~nm})$, however, only small differences between the IPCE curves are visible. This is in accordance with the assumption that less excitons generated within the DBP layer are quenched at the HIL1.3 buffer layer, but instead dissociate at the $\mathrm{DBP} / \mathrm{C}_{60}$ interface, generating free charge carriers.

$\mathrm{N}, \mathrm{N}^{\prime}$-bis(naphthalen-1-yl)-N,N'-bis(phenyl)-2,2'-dimethylbenzidine $(\alpha-\mathrm{NPD})$ also fulfills the requirements for efficient blocking layers in combination with DBP concerning energy level alignment and hole transporting ability, while hardly absorbing itself in the visible range. In contrast to highly crystalline DIP, thermally evaporated $\alpha$-NPD forms amorphous thin films. ${ }^{19}$ As there is no template effect for DIP, a similar gain in $J_{\mathrm{SC}}$ for amorphous blockers is expected. This assumption can be verified by the $J$ - $V$-characteristics (Fig. $3(\mathrm{a}))$. For the best cell with a $9 \mathrm{~nm}$ thick $\alpha$-NPD layer, $J_{\mathrm{SC}}$ increases by $29 \%$. Compared to the $30 \%$ of the device exhibiting $6 \mathrm{~nm}$ DIP, one can state that there is no difference in $J_{\mathrm{SC}}$ between devices with crystalline or amorphous blocking layers within the range of error. Moreover, the same trends for $V_{\mathrm{OC}}$ and FF can be observed compared to devices with crystalline blocking layer (Fig. 3(c)), so that there is again an increase in PCE of about 37\%. This leads to the conclusion that a possible template effect of crystalline blocking layers as proposed in literature ${ }^{5}$ is not occurring or at least its impact is negligible. However, there is a big difference in the thickness dependence of device parameters between these two blocking layers. While there was hardly any correlation between layer thickness and device performance for the DIP-containing solar cells, this is not the case for the $\alpha$-NPD devices. This is connected with two factors. First, it is assumed that 3 and even $6 \mathrm{~nm}$ of $\alpha$-NPD are not sufficient to form a closed layer, which leads to incomplete blocking and thus less gain in $J_{\mathrm{SC}}$ compared to structurally identical DIP devices. Second, blocking layers exceeding $9 \mathrm{~nm}$ show an increasing s-shape behavior.

We ascribe this feature to a growing transport resistance, ${ }^{20}$ an effect which is naturally much more pronounced for amorphous films as they generally feature lower charge carrier mobilities. To confirm, samples with $21 \mathrm{~nm} \alpha$-NPD highly doped with $\mathrm{MoO}_{x}$ (9:1 and 4:1) were prepared. As a result, the s-shape vanishes (open symbols in Fig. 3(a)). However, as $\mathrm{MoO}_{x}$ also acts as a quencher, $J_{\mathrm{SC}}$ decreases


FIG. 3. (a) $J-V$-characteristics, (b) IPCE curves and (c) solar cell parameters vs thickness of the $\alpha$-NPD blocking layer. The architecture of the PHJ solar cells is $\operatorname{ITO}(140 \mathrm{~nm}) / \mathrm{HIL} 1.3(45 \mathrm{~nm}) / \alpha-\mathrm{NPD}(\mathrm{x} \mathrm{nm}) / \mathrm{DBP}(15 \mathrm{~nm}) / \mathrm{C}_{60}(45 \mathrm{~nm}) /$ $\mathrm{BCP}(5 \mathrm{~nm}) / \mathrm{Al}(100 \mathrm{~nm})$. Again, for reasons of clarity, some curves were omitted in (a) and (b).

again with increasing percentage of $\mathrm{MoO}_{x}$. The quenching effect is also revealed by the corresponding IPCE characteristics (open symbols in Fig. 3(b)). IPCE curves naturally show the same trend as $J_{\mathrm{SC}}$ with an increasing amount of generated charge carriers up to a layer thickness of $9 \mathrm{~nm}$ and a following saturation for thicker blocking layers.

Mixing donor and acceptor molecules to enhance their interface resulting in more efficient exciton dissociation is a well-established concept. ${ }^{21,22}$ In this work, so-called planarmixed heterojunction devices were prepared, a combination of strictly planar and bulk heterojunction devices, combining the benefits of both concepts. ${ }^{23}$ This means that a mixed layer of DBP and $\mathrm{C}_{60}$ is sandwiched between a DBP layer on the anode and a $\mathrm{C}_{60}$ layer on the cathode side. Furthermore, devices with added blocker layer and skipping the pure DBP layer were fabricated. The volume ratio DBP:fullerene in the 
bulk was chosen $1: 2$, as this composition has proven to provide high PCEs for this material combination. ${ }^{24}$

In contrast to PHJ devices, there are significant differences in $J$ - $V$-characteristics comparing crystalline DIP and amorphous $\alpha$-NPD as blocking layers (Figure 4(a)). As the main difference concerns $V_{\mathrm{OC}}$, this is not related to the morphology of the blockers but can be associated to shifting energy levels and a change of the effective work function of the anode. Compared to PHJ devices, already the $V_{\mathrm{OC}}$ of the reference PM-HJ cell without blocker drops from $0.90 \mathrm{~V}$ to $0.84 \mathrm{~V}$, which declines further to $0.79 \mathrm{~V}$ introducing a DIP layer and even to $0.76 \mathrm{~V}$ by additionally skipping the pure DBP layer. The opposite effect is observed inserting $\alpha$-NPD. In these cells, $V_{\mathrm{OC}}$ increases to the value of the PHJ cell, lying a little bit higher for the cell without the neat DBP layer.

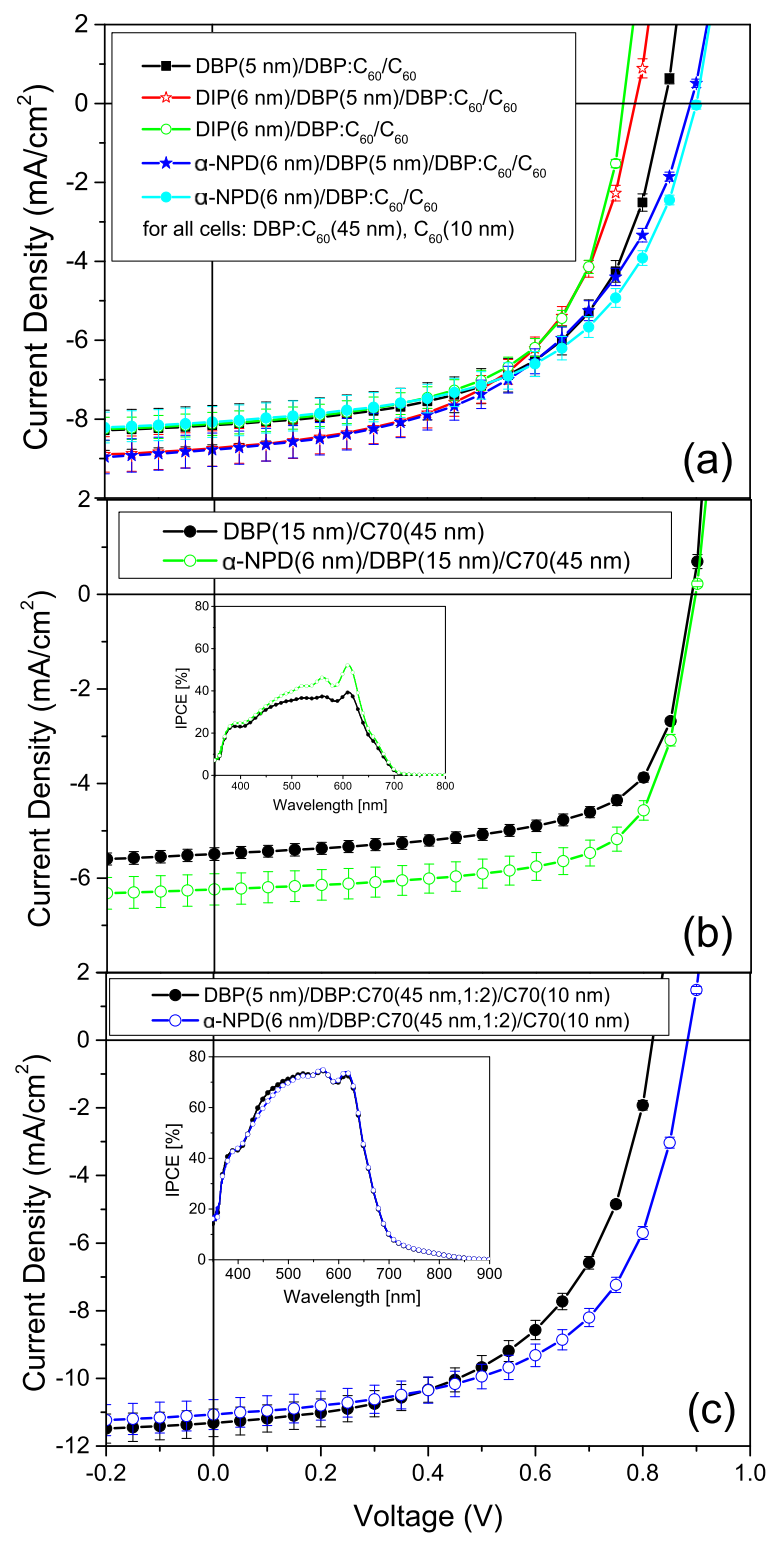

FIG. 4. $J$ - $V$-characteristics of (a) DBP: $\mathrm{C}_{60} \mathrm{PM}-\mathrm{HJ}$ devices (volume ratio 1:2), (b) $\mathrm{DBP} / \mathrm{C}_{70} \mathrm{PHJ}$ devices, and (c) $\mathrm{DBP}: \mathrm{C}_{70} \mathrm{PM}-\mathrm{HJ}$ solar cells. The insets in (b) and (c) show the related IPCE curves. The architecture of the solar cells is $\operatorname{ITO}(140 \mathrm{~nm}) / \mathrm{HIL} 1.3(45 \mathrm{~nm}) /$ organic/BCP $(5 \mathrm{~nm}) / \mathrm{Al}(100 \mathrm{~nm})$, with the active organic layers explained within each diagram.
For $J_{\mathrm{SC}}$ again there is an increase upon introduction of the blocker layer, however, it is smaller compared to that in PHJ devices. This is explained by the more efficient exciton dissociation already given by the device architecture, leading to less excitons reaching the blocking layer interface. Despite that gain in $J_{\mathrm{SC}}$ there is hardly any rise in PCE as the cells showing a higher current either lack in $V_{\mathrm{OC}}$ (DIP, open stars) or in FF ( $\alpha$-NPD, filled stars). The cells without the pure DBP layers show the same $J_{\mathrm{SC}}$ as the reference. This is due to the fact that blocking excitons with DIP or $\alpha$-NPD compensates additional absorption of the thin DBP layer. Thus, the best cell in this series is the $\alpha-\mathrm{NPD} / \mathrm{DBP}: \mathrm{C}_{60} / \mathrm{C}_{60}$ device, showing a small increase in PCE from $3.9 \%$ of the cell without blocking layer to $4.0 \%$, mainly due to the gain in $V_{\mathrm{OC}}$.

Naturally, there is a close connection between the choice of the acceptor and the blocking layer beneath the cathode (e.g., BCP), as excitons which are created within the acceptor can be blocked at the common interface. Although no common interface between acceptor and blocking layer at the anode exists, the choice of the acceptor material is also of importance for the strength of the blocking effect at the anode interface. This can be explained by looking at the IPCE characteristics. For PHJ devices, substituting $\mathrm{C}_{60}$ with $\mathrm{C}_{70}$ the absorption characteristic changes completely and thus the IPCE (inset Fig. 4(b)). As $\mathrm{C}_{70}$ also strongly absorbs in the same region as DBP does, there is redistribution in absorption appearing in the IPCE curves, meaning that less excitons are generated within the DBP, while parasitic absorption occurs within the acceptor. As a consequence, the gain in $J_{\mathrm{SC}}$ by introducing blocking layers beneath the donor is only half as big as in the case of $\mathrm{C}_{60}$ as acceptor (Fig. 4(b)). Hence, as the small increase in $V_{\mathrm{OC}}$ and FF is retained for this material combination, we got an increase in PCE of $19 \%$. This result shows that the success of introducing blocking layers at the anode interface depends strongly on the choice of materials. The more absorption occurs in the donor, the more gain in PCE can be achieved.

While the gain in PCE for PHJ devices is decreased using $\mathrm{C}_{70}$ instead of $\mathrm{C}_{60}$, it is just vice versa for PM-HJ solar cells (Fig. 4(c)). However, this is not explained by blocking reasons and therefore a higher gain in $J_{\mathrm{SC}}$. On the contrary, compared to the reference without blocker (filled circles) even a small decrease in $J_{\mathrm{SC}}$ is observed. Though, this deficit is easily compensated by an increase of fill factor from 56\% to $60 \%$. Due to the gain in $V_{\mathrm{OC}}$ an increase in PCE of $12 \%$ is reached leading to an overall efficiency of $5.8 \% \pm 0.2 \%$. Thus, for small molecule organic photovoltaic cells we could show that-depending on the choice of the buffer layer and the D/A combination-also for PM-HJ solar cells a remarkable efficiency enhancement is possible, introducing suitable blocking layers at the anode interface.

This work was supported by the German Research Foundation (DFG) within the priority program SPP 1355 ("Elementary Processes of Organic Photovoltaics"), by the Bavarian State Ministry of Science, Research and the Arts within the collaborative research network "Solar Technologies go Hybrid" and the Bavaria California Technology Center (BaCaTeC). S.G. and U.H. acknowledge the Bavarian Research Foundation for Ph.D. scholarships. 
${ }^{1}$ P. Peumans, V. Bulovic, and S. R. Forrest, Appl. Phys. Lett. 76, 2650 (2000). ${ }^{2}$ H. Gommans, B. Verreet, B. P. Rand, R. Muller, J. Poortmans, P. Heremans, and J. Genoe, Adv. Funct. Mater. 18, 3686 (2008).

${ }^{3}$ S. Naka, H. Okada, H. Onnagawa, and T. Tsutsui, Appl. Phys. Lett. 76, 197 (2000).

${ }^{4}$ A. Steindamm, M. Brendel, A. K. Topczak, and J. Pflaum, Appl. Phys. Lett. 101, 143302 (2012).

${ }^{5}$ Y. Zhou, T. Taima, T. Kuwabara, and K. Takahashi, Adv. Mater. 25, 6069 (2013).

${ }^{6}$ M. Hirade and C. Adachi, Appl. Phys. Lett. 99, 153302 (2011).

${ }^{7}$ D. Fujishima, H. Kanno, T. Kinoshita, E. Maruyama, M. Tanaka, M. Shirakawa, and K. Shibata, Sol. Energy Mater. Sol. Cells 93, 1029 (2009).

${ }^{8}$ X. Xiao, K. J. Bergemann, J. D. Zimmerman, K. Lee, and S. R. Forrest, "Small-Molecule Planar-Mixed Heterojunction Photovoltaic Cells with Fullerene-Based Electron Filtering Buffers," Adv. Energy Mater. (published online).

${ }^{9}$ D. Yokoyama, Z. Q. Wang, Y.-J. Pu, K. Kobayashi, J. Kido, and Z. Hong, Sol. Energy Mater. Sol. Cells 98, 472 (2012).

${ }^{10}$ D.-H. Lee, Y.-P. Liu, K.-H. Lee, H. Chae, and S. M. Cho, Org. Electron. 11, 427 (2010).

${ }^{11}$ A. Wilke, J. Endres, U. Hörmann, J. Niederhausen, R. Schlesinger, J. Frisch, P. Amsalem, J. Wagner, M. Gruber, A. Opitz, A. Vollmer, W. Brütting, A. Kahn, and N. Koch, Appl. Phys. Lett. 101, 233301 (2012).

${ }^{12}$ A. C. Dürr, F. Schreiber, M. Münch, N. Karl, B. Krause, V. Kruppa, and H. Dosch, Appl. Phys. Lett. 81, 2276 (2002).
${ }^{13}$ D. Kurrle and J. Pflaum, Appl. Phys. Lett. 92, 133306 (2008).

${ }^{14}$ J. Wagner, M. Gruber, A. Hinderhofer, A. Wilke, B. Bröker, J. Frisch, P. Amsalem, A. Vollmer, A. Opitz, N. Koch, F. Schreiber, and W. Brütting, Adv. Funct. Mater. 20, 4295 (2010).

${ }^{15}$ S. Kowarik, A. Gerlach, S. Sellner, F. Schreiber, L. Cavalcanti, and O. Konovalov, Phys. Rev. Lett. 96, 125504 (2006).

${ }^{16}$ M. B. Casu, S.-A. Savu, B.-E. Schuster, I. Biswas, C. Raisch, H. Marchetto, T. Schmidt, and T. Chasse, Chem. Commun. 48, 6957 (2012).

${ }^{17}$ K. Cnops, B. P. Rand, D. Cheyns, and P. Heremans, Appl. Phys. Lett. 101, 143301 (2012).

${ }^{18}$ M. Gruber, M. Rawolle, J. Wagner, D. Magerl, U. Hörmann, J. Perlich, S. V. Roth, A. Opitz, F. Schreiber, P. Müller-Buschbaum, and W. Brütting, Adv. Energy Mater. 3, 1075 (2013).

${ }^{19}$ N. Koch, A. Elschner, J. Schwartz, and A. Kahn, Appl. Phys. Lett. 82, 2281 (2003).

${ }^{20}$ J. Wagner, M. Gruber, A. Wilke, Y. Tanaka, K. Topczak, A. Steindamm, U. Hörmann, A. Opitz, Y. Nakayama, H. Ishii, J. Pflaum, N. Koch, and W. Brütting, J. Appl. Phys. 111, 054509 (2012).

${ }^{21}$ J. J. M. Halls, C. A. Walsh, N. Greenham, E. A. Marseglia, R. Friend, S. C. Moratti, and A. Holmes, Nature 376, 498 (1995).

${ }^{22}$ G. Yu, J. Gao, J. C. Hummelen, F. Wudl, and A. J. Heeger, Science 270, 1789 (1995).

${ }^{23}$ J. Xue, B. Rand, S. Uchida, and S. Forrest, Adv. Mater. 17, 66 (2005).

${ }^{24}$ Z. Wang, D. Yokoyama, X.-F. Wang, Z. Hong, Y. Yang, and J. Kido, Energy Environ. Sci. 6, 249 (2013). 